\begin{tabular}{|c|l|}
\hline Title & Surface flatness of polycrystall line copper after argon ion etching followed by annealing \\
\hline Author(s) & Hino, T.; Taguchi, T.; Y amauchi, Y.; Hirohata, Y.; Nishikawa, M. \\
\hline Citation & $\begin{array}{l}\text { Journal of Vacuum Science \& Technology B: Microelectronics and Nanometer Structures, 22(6), 2632-2634 } \\
\text { https:/doi.org/10.1116/1.1814110 }\end{array}$ \\
\hline Issue Date & 2004 11-04 \\
\hline Doc URL & http://hdl.handle.net/2115/5710 \\
\hline Type & article (author version) \\
\hline File Information & JV STB22-6.pdf \\
\hline
\end{tabular}

Instructions for use 


\title{
Surface flatness of polycrystalline copper after argon ion etching followed by annealing
}

\author{
T. Hino, ${ }^{\text {a) }}$ T. Taguchi, Y. Yamauchi, and Y. Hirohata \\ Laboratory of Plasma Physics and Engineering, Hokkaido University, Kita-13, Nishi-8, Kita-ku, \\ Sapporo 060-8628, Japan \\ M. Nishikawa \\ Science Solutions International Laboratory, Inc., 21-7, Naka-machi, Meguro-ku, Tokyo 153-0065, Japan
}

(Received 18 June 2004; accepted 13 September 2004; published 4 November 2004)

\begin{abstract}
Oblique injection of argon ions with energy of $1 \mathrm{keV}$ was conducted for etching of polycrystalline copper. The surface became rough owing to the formation of blisters. The average diameter and height of blisters was approximately 150 and $20-30 \mathrm{~nm}$, respectively. The polycrystalline copper irradiated by argon ions was annealed to rupture the blisters. The retained argon desorbed in the temperature range from 500 to $800 \mathrm{~K}$. The height of blisters significantly reduced to $10-15 \mathrm{~nm}$ although the diameter and surface density of blisters roughly remained the same. () 2004 American Vacuum Society. [DOI: 10.1116/1.1814110]
\end{abstract}

\section{INTRODUCTION}

Polycrystalline copper has been widely employed as a wiring material for large integrated circuits (LSI). For miniaturized and/or multilayer LSI, the flatness of the wiring has to be improved compared with the product of present etching methods such as chemical mechanical polishing (CMP). ${ }^{1}$ In CMP, the smoothness obtained is $50-100 \mathrm{~nm}$. The production processes of LSI may be simplified if a dry processing is applied to improve the smoothness for the etching of polycrystalline copper, instead of the present wet processing. In the case of ion etching based on physical sputtering, the surface becomes rough owing to the formation of blisters. This roughness has to be smaller than that owing to the wet processing. In the previous experiment, ${ }^{2}$ oblique argon ion injection for the copper surface was conducted and it was found that the surface flatness was improved by the oblique ion injection, compared with the case of perpendicular ion injection, since the top parts of blisters were selectively etched. After the oblique ion injection, the surface roughness owing to blisters with a height of approximately $20 \mathrm{~nm}$ was observed. This height is smaller than that of the wet processing. In the present experiment, in order to further improve the smoothness, an annealing was conducted to rupture the blisters and reduce the size of blisters for the polycrystalline copper irradiated by oblique argon ion injection. The surface flatness after the annealing was compared with that before the annealing.

\section{EXPERIMENT AND RESULT}

A polycrystalline copper sample was mechanically polished and cleaned in an ultrasonic bath. The sample size was $100 \times 100 \times 0.1 \mathrm{~mm}$. The grain size was approximately 100 $\mathrm{nm}$. The sample was placed in the argon ion irradiation apparatus, AES apparatus, shown in Fig. 1, and irradiated by

a) Also at Science Solutions International Inc., 21-7, Naka-machi, Meguro-ku 153-0065, Japan; electronic mail: tomhino@qe.eng.hokudai.ac.jp argon ions at different incident angles. The temperature of the sample was room temperature (RT) and the energy of argon ion was $1 \mathrm{keV}$. The flux of argon ions was measured by the sample current, and the value was approximately $10^{14} \mathrm{Ar} / \mathrm{cm}^{2} \mathrm{~s}$. The ion fluence was taken (0.9-1.2) $\times 10^{17} \mathrm{Ar} / \mathrm{cm}^{2}$ for the weight loss to be in the range from 16 to $20 \mu \mathrm{g} / \mathrm{cm}^{2}$. The etching time was $1000 \mathrm{~s}$ for a fluence of $10^{17} \mathrm{Ar} / \mathrm{cm}^{2}$. The etching depth corresponding to the fluence is approximately $25 \mathrm{~nm}$. After the ion irradiation, the surface morphology was observed by using atomic force microscope (AFM, SHIMADZU SPM-9500). The morphology was taken by using a contact mode. The left-hand side of Fig. 2 shows the surface morphologies after the ion irradiation at different angles. In the case of perpendicular injection with an incident angle of $0^{\circ}$ the average diameter of blisters was large, $170 \mathrm{~nm}$, but the surface density of blisters was low, 1.3 $\times 10^{9} / \mathrm{cm}^{2}$. The average height of blisters was also large, 30 $\mathrm{nm}$. As the incident angle was increased, the average diameter decreased to $125 \mathrm{~nm}$ and the surface density increased up to $4 \times 10^{9} / \mathrm{cm}^{2}$. The height of blisters decreased to approximately $20 \mathrm{~nm}$. This result shows that the top parts of blisters are selectively etched by the oblique ion injection and the use of oblique ion injection is useful to reduce the height and diameter of blisters. The size of blisters observed in the above-mentioned experiment was roughly the same as the grain size.

The samples irradiated by argon ions shown on the lefthand side of Fig. 2 were heated from RT to $1000 \mathrm{~K}$ in an apparatus of thermal desorption spectroscopy. ${ }^{3}$ The sample was heated by an infrared furnace and the desorption rate of argon atoms was quantitatively measured by using a quadruple mass spectrometer. The heating rate was $0.5 \mathrm{~K} / \mathrm{s}$ and the temperature was linearly increased. After the heating, the change of surface morphology was observed. The right hand side of Fig. 2 shows the surface morphologies after the heating. Although the average diameter and density of blisters roughly remained the same, the height of blisters clearly decreased as shown in Fig. 3. In particular, the reduction of the 


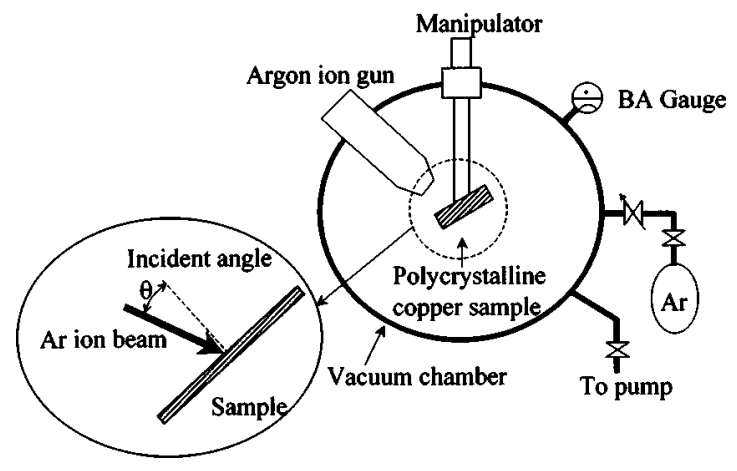

FIG. 1. Apparatus of argon ion irradiation.

height was largest in the case of the perpendicular ion injection. In this case, the height of blisters decreased from 30 to $10 \mathrm{~nm}$. The decrease of the height in the cases of oblique ion injection was approximately $30 \%$ of the value before the heating. The height in the case of oblique ion injection was in the range from 11 to $15 \mathrm{~nm}$. A similar experiment was carried out by Ritter et al. ${ }^{4}$ for $\mathrm{Cu}(001)$ surface, using 400 $\mathrm{eV}$ argon ion irradiation. In this experiment, the fluence was low, $10^{15} \mathrm{Ar} / \mathrm{cm}^{2}$, so that only the damage structure called vacancy islands was observed. The annealing flattened the rough surface due to the damage. In our experiment, the fluence was taken high, $10^{17} \mathrm{Ar} / \mathrm{cm}^{2}$, for the purpose of etching, and thus the blisters were formed. The annealing also flattened the roughness owing to the blisters.

The thermal desorption spectra of argon were taken for the samples irradiated by argon ions at different angles. Figure 4 shows the thermal desorption spectra for the cases of perpendicular ion injection and oblique ion injection with incident angle of $45^{\circ}$. The spectral shapes at $22.5^{\circ}$ and $67.5^{\circ}$ were similar to those at $0^{\circ}$ and $45^{\circ}$, respectively. The retained

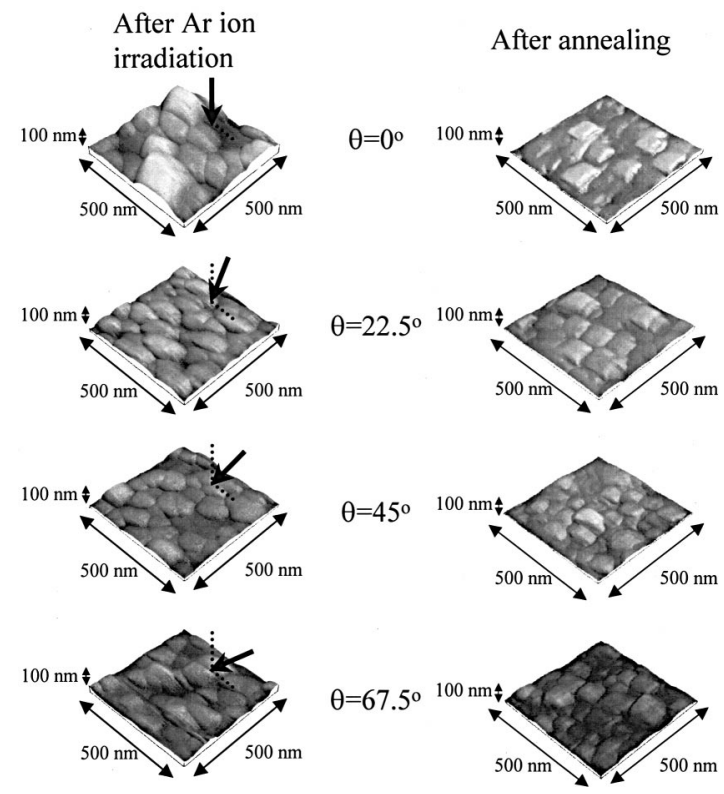

FIG. 2. Surface morphologies of polycrystalline copper after oblique injection of argon ion with different angle (left) and after annealing (right).

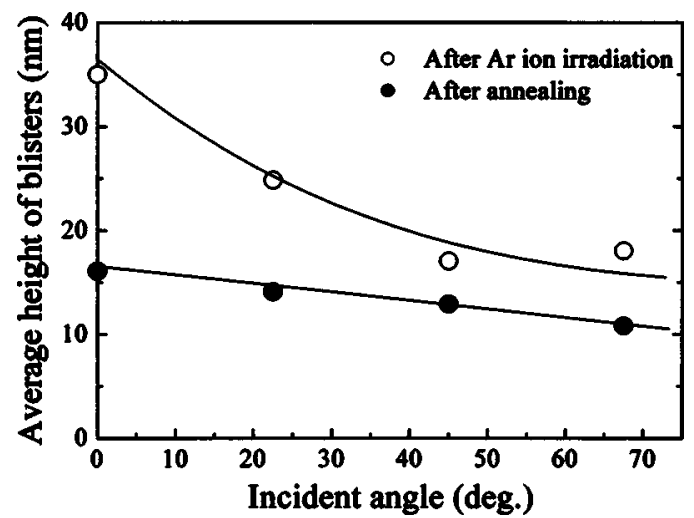

FIG. 3. Height of blisters before and after annealing vs incident angle.

argon desorbed in the temperature range from 500 to $800 \mathrm{~K}$. The fractions of retained argon were $1 \%, 0.6 \%, 0.3 \%$, and $0.3 \%$ of the incident argon fluence, respectively. The retained amount of argon decreased while the incident angle was increased, since the implantation depth becomes shallow. The thermal desorption spectra clearly showed the retention of argon. Hence, this result suggests that the blisters and voids were produced at the present irradiation condition. In the previous experiment, ${ }^{2}$ the formation of blisters was observed for argon energy higher than $100 \mathrm{eV}$ when the fluence was $1.5 \times 10^{17} \mathrm{Ar} / \mathrm{cm}^{2}$. The thermal desorption spectra of Fig. 4 show that the ruptures of surface blisters and voids with argon atoms took place in the temperature range from 500 to $800 \mathrm{~K}$. Hence, annealing at a temperature of approximately $800 \mathrm{~K}$ is required to rupture the blisters.

A similar experiment using helium ions was conducted. ${ }^{5}$ The samples of polycrystalline copper irradiated by helium ions with energy of $5 \mathrm{keV}$ and different angles were annealed up to $1000 \mathrm{~K}$ and then the change of the surface morphologies was examined. The average diameter and surface density of blisters were $230-450 \mathrm{~nm}$ and $(3-9) \times 10^{8} / \mathrm{cm}^{2}$ in the case of helium ion injection. The average diameter of blisters was approximately twice larger than that of blisters resulting from argon ion injection although the density of blisters was a few times lower. The height of blisters was $40 \mathrm{~nm}$ in the case of helium ion injection. This value was several times

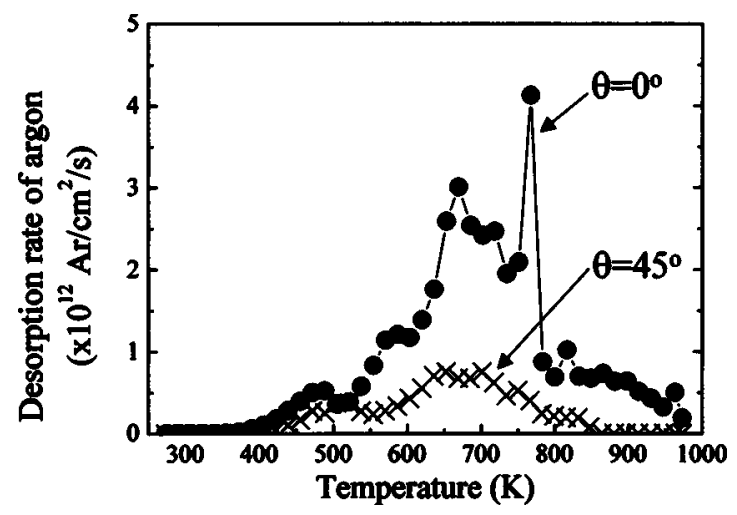

FIG. 4. Thermal desorption spectra of argon after argon ion injection with incident angles of $0^{\circ}$ and $45^{\circ}$ vs heating temperature. 
larger than the height produced by argon ion injection. The thermal desorption spectra showed the retained helium desorbed in the temperature range from 500 to $800 \mathrm{~K}$. The range of temperature for rupture of surface blisters and voids with helium atoms was roughly the same as the case of argon.

\section{CONCLUSION}

The present experiment showed that the roughness caused by argon ion irradiation owing to the formation of blisters was smoothened by annealing at a temperature of approximately $800 \mathrm{~K}$. The height of blisters was reduced to approximately $10 \mathrm{~nm}$. When the energy of argon ions was taken lower than $1 \mathrm{keV}$, the height of blisters became smaller than the present value. ${ }^{2}$ Hence, it may be possible to further improve the surface flatness if the polycrystalline copper is irradiated with argon ions having lower energy, and then annealed up to approximately $800 \mathrm{~K}$.

${ }^{1}$ T. Doi, Details Semiconductor CMP Technology (Kogyo Chousakai, Tokyo, 2001).

${ }^{2}$ T. Taguchi, Y. Yamauchi, Y. Hirohata, T. Hino, and M. Nishikawa, Appl. Surf. Sci. (to be published).

${ }^{3}$ T. Hino et al., Nucl. Fusion 44, 496 (2004).

${ }^{4}$ M. Ritter, M. Stindtmann, M. Farle, and K. Baberschke, Surf. Sci. 348, 243 (1996).

${ }^{5}$ T. Hino, T. Taguchi, Y. Yamauchi, Y. Hirohata, and M. Nishikawa, Appl. Surf. Sci. (to be published). 